\title{
Variações da artéria hepática: estudo anatômico em cadáveres
}

\section{Variations of hepatic artery: anatomical study on cadavers}

\author{
Geraldo Alberto Sebben, tCbC-PR'; Sérgio luiz Rocha, TCbC-PR2; Marco Auréllo Sebben³; Plácido Roberto Parussolo Fllho3; \\ Bruno Henrique Habu Gonçalves ${ }^{3}$
}

\section{R E S U M O}

\begin{abstract}
Objetivo: Demonstrar as minúcias do sistema arterial hepático, a incidência das variações anatômicas e comparar os dados obtidos com os da literatura. Métodos: Foram preparados 45 cadáveres do Departamento de Anatomia da Pontifícia Universidade Católica do Paraná, entre julho de 2010 e abril de 2011, sendo aproveitados 30 que possuíam integridade das estruturas. Analisaram-se as variações anatômicas das artérias hepáticas, suas principais características, como origem, trajeto, comprimento e diâmetro. O resultado global foi expresso por frequência e percentual de cadáveres com variações anatômicas do sistema arterial hepático. A estimativa deste percentual foi feita construindo-se um intervalo de confiança de $95 \%$. Resultados: Observou-se algum tipo de variação anatômica em $40 \%(n=12)$ dos cadáveres estudados. Encontraram-se variações em duas artérias hepáticas comuns, três artérias gastroduodenais, três artérias hepáticas direita, uma artéria hepática esquerda, uma artéria gástrica direita e duas artérias císticas. Quanto ao tronco celíaco, verificaram-se variações em seu comprimento, diâmetro e altura de sua origem que foi comum na aorta. A variação da artéria hepática direita originando-se da artéria mesentérica superior foi encontrada em $10 \%(n=3)$ dos espécimes estudados e foi considerado o tipo de variação mais prevalente neste estudo. Conclusão: As variações nas artérias hepáticas são encontradas com frequência, e neste estudo foi $40 \%$, valor semelhante ao da literatura. A variação mais significativa, observada em 10\% (3 casos), foi a da artéria hepática direita com sua origem na artéria mesentérica superior.
\end{abstract}

Descritores: Artéria hepática. Fígado. Cirurgia geral. Anatomia. Cadáver.

\section{INTRODUÇÃO}

O conhecimento anatômico do corpo humano data de 500 anos antes de Cristo, no sul da Itália, com Alcméon de Crotona, que realizou as primeiras dissecações, mesmo que em animais. No Século III a.C., o estudo da anatomia avançou consideravelmente em Alexandria e muitas descobertas lá realizadas podem ser atribuídas a Herófilo e Erasístrato, os primeiros a realizar dissecações humanas de modo sistemático.

Galeno foi o primeiro anatomista a analisar detalhadamente o sistema arterial proveniente do tronco celíaco, descrevendo que as artérias destinadas ao estômago, fígado e baço não nasciam em tronco comum na aorta como a artéria destinada ao intestino, mas, ao contrário, em dois troncos distintos. Andréas Vesalius, no Século XVI, proporcionou descrições anatômicas superiores às de Galeno, comentando sobre a divisão em dois ramos de tronco celíaco: direito, correspondente à artéria hepáti$\mathrm{ca}$, e esquerdo, correspondente à artéria esplênica que forneceria ramo gástrico a artéria gástrica esquerda. No entanto, Jacques Benigne Winslow e Albert Haller, consi- derados os pais da angiologia moderna, definiram corretamente a anatomia do tronco celíaco. Winslow descreveu completamente o tronco e seus ramos e Haller abordou os detalhes anatômicos anômalos da artéria hepática ${ }^{1}$.

Conforme descrito por ilustres anatomistas, como Testut, Moore, Sobotta e Netter, a anatomia da artéria hepática se dá quando o tronco celíaco com origem na aorta ramifica-se em artéria gástrica esquerda, artéria esplênica e artéria hepática comum. Esta última, após a emergência da artéria gastroduodenal, continua-se como artéria hepática própria e ramifica-se em artéria hepática direita e esquerda no hilo hepático ${ }^{2-5}$. Esta configuração é a adotada pela maioria dos estudiosos do tema, destacamos Michels et al. e Soin et al. ${ }^{6,7}$. Segundo a literatura, variações neste sistema arterial ocorrem aproximadamente entre 25 e $42 \%$. Bertevello et al. e Soares et al. descreveram ainda casos raros de variações, dentre eles citamos artéria hepática dupla; artéria hepática direita da artéria mesentérica inferior; artéria hepática passando por trás da veia porta ${ }^{8,9}$.

Diante da alta incidência das variações no sistema arterial hepático e sua influência nos procedimentos

Trabalho realizado no Departamento de Anatomia da Pontifícia Universidade Católica do Paraná- PR-BR.

1. Professor de Anatomia Médica da PUCPR, Chefe do Departamento de Cirurgia Geral e da Residência Médica do Hospital São Vicente de Curitiba; 2. Professor de Anatomia Médica da PUCPR e da UFPR; 3. Acadêmico do Curso de Medicina da PUCPR. 
que envolvem a região, é de suma importância que ela seja bem estudada, detalhada e conhecida por estudantes e profissionais da área de saúde. Este conhecimento é relevante para as cirurgias hepáticas principalmente o transplante. Um número expressivo de complicações pode ser evitado quando do reconhecimento de possíveis variações anatômicas, tanto na captação quanto no implante hepático de cadáveres ou intervivos.

Neste contexto o estudo e a aprendizagem da multiplicidade das variações anatômicas, em especial as do fígado, são sempre necessárias e fundamentais. Reconhecê-las e manejá-las adequadamente é muito importante para o sucesso diagnóstico e da terapia cirúrgica.

O objetivo deste estudo foi dissecar e estudar em cadáveres humanos as minúcias do sistema arterial hepático, a incidência das variações anatômicas e comparar os dados obtidos com os da literatura, contribuindo, desta forma, com estudantes e profissionais que atuam nesta área.

\section{MÉTODOS}

Foram dissecados e analisados 45 cadáveres no laboratório do Departamento de Anatomia da Faculdade de Medicina da Pontifícia Universidade Católica do Paraná. Foram descartados os espécimes que não possuíam integridade de sua estrutura física.

Os cadáveres humanos utilizados no estudo eram de ambos os sexos e diversas etnias, fixados e mantidos há mais de um ano em solução formal a $10 \%$. O material foi usado de acordo com a Lei 8501, de 30 de novembro de 1992, que dispõe sobre a utilização de cadáveres não reclamados para fins de estudo ou pesquisa científica.

Este estudo foi apresentado ao Comitê de Ética em Pesquisa da PUCPR e aprovado sob o parecer $n^{\circ}$ 0005071/11.

Utilizou-se neste estudo uma amostra constituída por 30 cadáveres mestiços, de idade aproximada variando entre 20 e 70 anos. Alguns espécimes anatômicos eram pré-dissecados, sendo $83,33 \%(n=25)$ do sexo masculino e $16,66 \%(n=5)$ do sexo feminino. Analisou-se a origem, posição, trajeto e aferiu-se comprimento e diâmetro das artérias, bem como, as relações de todas as artérias provenientes do tronco celíaco, dando ênfase, porém, às do sistema arterial hepático.

A fim de facilitar o estudo e a comparação dos dados, criamos uma tabela que foi usada para a coleta de dados. Marcamos as artérias estudadas com fios coloridos, a fim de diferenciar artérias, veias e os ductos da via biliar (Figura 1). Os achados foram documentados fotograficamente e os dados foram tabelados e comparados com os descritos da literatura.

Concomitantemente à coleta dos dados na pesquisa de campo, bem como, a realização inicial desta, realizamos uma vasta revisão de literatura, pois todos os dados coletados foram comparados com vários autores. A revisão foi realizada nas bases de dados disponíveis pela Pontifícia Universidade Católica do Paraná em seus Laboratórios de Informática e Biblioteca Central.

Quanto à análise estatística, o resultado global foi expresso por frequência e percentual de cadáveres com variações anatômicas do sistema arterial hepático. A estimativa deste percentual foi feita construindo-se um intervalo de confiança de $95 \%$.

Com a pesquisa de campo foram obtidos os dados e as características relativas ao sistema arterial hepático. Anotamos o comprimento, diâmetro e origem de todas as artérias provenientes do tronco celíaco e algumas importantes variações anatômicas. Os dados obtidos e analisados são abaixo reproduzidos.

\section{RESULTADOS}

Visualizou-se algum tipo de variação anatômica, considerando-se desde o tronco celíaco, artérias hepáticas comum, própria, direita, esquerda, gastroduodenal, gástrica direita e cística, em 40\% ( $n=12)$ dos 30 cadáveres dissecados. Analisando-se estatisticamente o resultado global pela frequência e percentual de cadáveres com variações anatômicas das artérias hepáticas e construindo-se um intervalo de confiança de $95 \%$, existe, portanto, a chance de que o intervalo de $22,5 \%$ a $57,5 \%$ contenha o verdadeiro percentual de cadáveres com variações.

As variações ocorreram em três dos cadáveres do sexo feminino e em nove dos cadáveres do sexo masculino. As variações anatômicas prevaleceram nos caucasianos $, 42,8 \%$, incidiram em $40 \%$ dos pardos e em $17,2 \%$ dos negros.

Todos os troncos celíacos estudados eram provenientes da aorta abdominal. As variações encontradas foram em seus ramos (Tabela 1). O comprimento médio do tronco celíaco, aferido de sua base até sua trifurcação, foi de $0,68 \mathrm{~cm}$. Um dos cadáveres diferiu acentuadamente do conjunto com um tronco celíaco de $2,9 \mathrm{~cm}$ de comprimento. Com relação ao diâmetro, a média foi $1,33 \mathrm{~cm}$, variando de 0,4 a $2 \mathrm{~cm}$.

Duas artérias hepáticas comuns apresentaram alteração. Elas eram originárias diretamente da artéria mesentérica superior. O comprimento médio encontrado nas artérias hepáticas comum foi $2,85 \mathrm{~cm}$, variando de 0,6 a 4,7. O diâmetro médio foi 0,68 variando de 0,5 a $1,2 \mathrm{~cm}$.

Encontrou-se alteração em três artérias gastroduodenais e em um cadáver a artéria gastroduodenal não foi identificada. Dentre as variações encontramos: duas da artéria hepática direita e outra da artéria mesentérica superior. Todas as outras eram provenientes da artéria hepática comum. O comprimento não foi identificado por dificuldades técnicas. O diâmetro médio encontrado foi 0,464, variando de 0,3 a $0,7 \mathrm{~cm}$. 
Considerada a partir do momento em que a artéria gastroduodenal se origina na artéria hepática comum, a artéria hepática própria foi considerada dentro dos padrões em 26 cadáveres. Ou seja, 13,3\% de variações. O comprimento médio encontrado nas artérias hepáticas comuns foi de $2,42 \mathrm{~cm}$, variando de 0,5 a 4,5 e seu diâmetro médio foi de 0,62 variando de 0,4 a 1,3cm.

Encontrou-se, em três casos, a artéria hepática direita originando-se da artéria mesentérica superior, corresponde a $10 \%$ dos cadáveres e peças com variações anatômicas. Destes, todos eram do sexo masculino, dois eram negros e um pardo. O comprimento médio foi 3,29 variando de 0,5 a 5,5 e seu diâmetro médio foi 0,5 variando de 0,2 a $0,8 \mathrm{~cm}$.

Com relação à artéria hepática esquerda, em um caso ela era originária diretamente da artéria mesentérica superior. O comprimento médio foi 2,46 variando de 0,6 a 4,7 e seu diâmetro médio foi 0,42 variando de 0,3 a $0,7 \mathrm{~cm}$.

Encontrou-se artéria cística variante em dois cadáveres e em um deles não foi identificada. Em um dos casos a artéria cística tinha sua origem na bifurcação das artérias hepática direita e esquerda e em outro caso na artéria hepática esquerda. O comprimento médio foi 2,45 variando de 0,1 a 4 e seu diâmetro médio foi 0,17 variando de 0,1 a $0,3 \mathrm{~cm}$.

Em 23 cadáveres, a artéria gástrica direita originava-se da artéria hepática própria. Em outros três, da artéria hepática comum. Encontrou-se um caso especial: a artéria gástrica direita era proveniente da artéria hepática esquerda. A artéria gástrica direita não foi identificada em três cadáveres.

\section{DISCUSSÃO}

O clássico estudo de Michels com 200 dissecções ${ }^{6}$, publicado em 1966, definiu as variações anatômicas básicas no fornecimento da artéria hepática e tem servido como referência para a grande maioria das subsequentes contribuições nesta área. Esta classificação envolve a artéria hepática comum, artéria hepática própria e as artérias hepática direita e esquerda; onde a Classe 1 corresponde à disposição normal do sistema arterial hepático; Classe 2 para a artéria hepática esquerda originando-se da artéria gástrica esquerda; Classe 3 para a artéria hepática direita originando-se da artéria mesentérica superior; Classe 4 para múltiplos ramos aberrantes; e Classe 5 para a artéria hepática comum originando-se da artéria mesentérica superior $^{10}$. Nosso trabalho seguiu esta classificação.

A análise de trabalhos que envolvem o sistema arterial hepático nos mostra que variações deste sistema podem ser encontradas entre $20 \%$ e $50 \%$ da população. Como exemplos, citamos trabalhos como o de Hiatt et al. que realizaram revisão da anatomia em 1000 pacientes submetidos a transplante de fígado e encontraram 24,3\% de alterações arteriais hepáticas ${ }^{11}$. Soin et al. encontraram $30,6 \%$ de anomalias em 527 doadores de fígado7; Chaib, $39 \%$ estudando 80 doadores $^{12}$. Michels, em sua série de

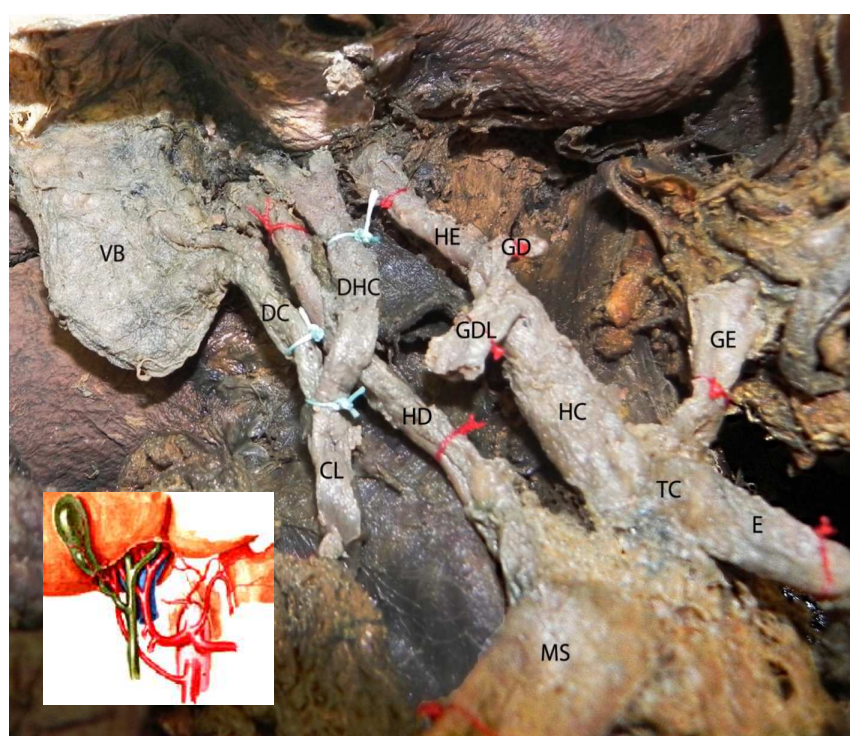

Figura 1 - Artéria Hepática Direita originando-se da Artéria Mesentérica Superior.

Legenda: MS: A. Mesentérica Superior; TC: Tronco Celíaco; GE: Artéria Gástrica Esquerda; E: A. Esplênica; HC: A. Hepática Comum; GD: A. Gástrica Direita; HE: A. Hepática Esquerda; GDL: A. Gastroduodenal, HD: A. Hepática Direita; VB: Vesícula Biliar; DC: Ducto Cístico; DHC. Ducto Hepático Comum; CL: Colédoco.

Tabela 1 - Variações anatômicas encontradas.

\begin{tabular}{lccc}
\hline Artéria & Origem & N & \% \\
\hline Hepática comum & Mesentérica superior & 2 & 6,66 \\
Gástrica direita & Hepática direita & 1 & 6,66 \\
Gástrica direita & Mesentérica superior & 3 & 3,33 \\
Hepática direita & Mesentérica superior & 1 & 10 \\
Hepática esquerda & Mesentérica superior & 1 & 3,33 \\
Cística & Bifurcação da hepática própria & 1 & 3,33 \\
Cística & Hepática esquerda & 3,33 \\
Gástrica direita & Hepática esquerda & 12 & 40 \\
Total & & 1 & \\
\hline
\end{tabular}


200 dissecções de cadáveres, mostrou $45 \%$ de anomalias anatômicas $^{10}$. O estudo de Kemeny et al. foi o trabalho que demonstrou o maior índice de variações, 50\%. Seu estudo foi realizado com arteriografias do tronco celíaco e artéria mesentérica superior em 100 pacientes $^{13}$. Em nosso estudo, o índice de variações encontradas na análise de 30 cadáveres e peças foi $40 \%(n=12)$, valor similar ao encontrado na maioria dos trabalhos citados.

Para a correta divisão da artéria hepática própria em hepática direita e esquerda (Classe 1) chegamos a um valor de $73,33 \%$. Na literatura os valores variam de $55 \%$ a $80 \%$. Destacamos Hiatt et al. com 75,7\% ${ }^{11}$.

A Classe 2, caracterizada pela artéria hepática esquerda originando-se da artéria gástrica esquerda, não foi encontrada em nosso estudo e a literatura nos mostra baixa prevalência da mesma. Bertevello e Chaib, que realizaram estudo em 60 fígados provenientes de cadáveres frescos, apresentaram apenas dois casos $(3,3 \%)$ desta variação $0^{8,12}$. Hiatt et al. obtiveram 9,7\% ${ }^{11}$.

Com relação à artéria hepática direita originando-se da artéria mesentérica superior (Classe 3), encontramos três casos (10\%) e foi a variação mais significativa apresentada. Na literatura os valores estão entre $8 \%$ e $18 \%$. Acreditamos que este tipo de variação apresente maior relevância tanto pela sua maior prevalência como pelo potencial de afetar procedimentos cirúrgicos. Nesta, pelo fato de a artéria correr entre a veia cava inferior e a veia porta, ao nível do triângulo hepatobiliar, deve-se ter muito cuidado em operações como as colecistectomias e as pancreatectomias. Destacamos assim a importância de sua identificação em procedimentos cirúrgicos.

A Classe 4 de Michels é destinada para casos que envolvem associação de artérias variantes, por exemplo artéria hepática direita proveniente da mesentérica superior e a hepática esquerda proveniente da gástrica esquerda. Trata-se de situações mais incomuns e não foi encontrado nenhum caso em nossa pesquisa. Hiatt encontrou alguma associação em apenas 2,3\% ${ }^{11}$.

A Classe 5 que se refere a artéria hepática comum originando-se da mesentérica superior foi encontrada em um cadáver (3,33\%). Daseler et al. realizaram estudo em que observaram 500 peças do sistema arterial hepático e observaram $4,4 \%$ de artéria hepática comum proveniente da artéria mesentérica superior ${ }^{14}$. Já Bertevello e Chaib encontraram esta variação em apenas um caso $(1,6 \%)^{8,12}$. Hiatt et al. encontrou esta variação em apenas $1,5 \%{ }^{11}$. Outro estudo realizado em 150 transplantes de fígado pelo serviço de transplante de fígado do Hospital de Clínicas da Universidade Federal do Paraná, Freitas et al. demostrou que a artéria hepática comum originava-se da mesentérica superior em quatro pacientes, totalizando $1,62 \%{ }^{15}$.

Dentre as variações descritas, algumas merecem destaque ainda maior por apresentarem maior risco de complicações em procedimentos cirúrgicos. Chaib descreveu artéria hepática cuja origem se fazia de tronco conectando tronco celíaco à artéria mesentérica superior, além de outro caso em que uma artéria hepática esquerda originava-se da artéria gástrica esquerda e ainda envolvia o esôfago ${ }^{12}$. Em nosso estudo merece destaque um cadáver $(3,33 \%)$ em que a artéria hepática comum originavase da artéria mesentérica superior, este quadro é classificado como Classe 5 de Michels. Quando a artéria hepática esquerda origina-se da artéria gástrica esquerda (Classe 2), existirá uma alta possibilidade comprometimento isquêmico do lóbulo esquerdo do fígado, se esta variação não for reconhecida durante uma operação de gastrectomia radical. Acreditamos que a variação do tipo 3, artéria hepática direita originando-se da mesentéria superior, apresente maior relevância pela sua maior prevalência.

O uso da tomografia helicoidal e arteriografia por tomografia tridimensional auxilia o cirurgião no planejamento da cirurgia hepática, em especial a do transplante, identificando de forma prévia o diâmetro da luz vascular, anomalias do suprimento arterial hepático, trombose de veia porta e aneurismas de artéria esplênica, já que a artéria esplênica pode ser local para anastomose vascular em casos de receptores com artérias hepáticas de diâmetro exíguo. Medidas como esta não são recomendadas em procedimentos menores, mas o cirurgião deve estar apto a identificar estas alterações no momento da operação.

O transplante de fígado foi introduzido por Starzl et al. e, desde então, muitas dificuldades foram superadas, porém a maior limitação para a sua aplicabilidade tem sido a falta de doadores de órgãos. Para minimizar a perda de órgãos, as artérias extra-hepáticas devem ser identificadas com precisão na ocasião da captação do órgão, evitando lesões que possam comprometer a completa arterialização do enxerto ${ }^{16}$.

Um fígado normal pode sobreviver à ligadura da artéria hepática devido à circulação portal e colateral. Ligaduras terapêuticas são realizadas abordando neoplasias hepáticas, traumas e lesões arteriais do fígado, conforme Suzuki et al. ${ }^{17}$. Tais procedimentos estão proscritos no paciente transplantado hepático, pelo fato de se seccionar todas as vias de circulação colateral do órgão na captação do enxerto, não se dispondo, desta maneira, do mecanismo que compensaria uma lesão do tronco arterial principal, fato que possibilitaria necrose do tecido com sérias consequências $^{15}$.

O estudo detalhado da anatomia das estruturas do fígado tanto intra como extra-hepáticas permitiu, nos últimos anos, o desenvolvimento com sucesso de técnicas alternativas de transplante hepático, como o transplante de fígado reduzido, chamado "split-liver" ou bipartição hepática e recentemente o transplante intervivos ${ }^{9}$. Em particular, na técnica de bipartição do fígado, com um fígado podemos obter dois enxertos (lobo direito e lobo esquerdo hepático), beneficiando, assim, dois receptores, técnica desenvolvida por Pilchlmayr et al. ${ }^{18}$. Segundo Couinaud e Houssin, é reduzido o número de casos (5\%) que apresentam algum tipo de variação anatômica que impossibilitari- 
am a bipartição do órgão para utilização no transplante de fígado por bipartição ${ }^{19}$. Portanto é de extrema importância um conhecimento adequado de todo sistema arterial hepático.

A precaução quando das intervenções cirúrgicas deve ser aplicada a diversos outros procedimentos, desde uma colecistectomia, gastrectomia até a pancreatectomia. Devemos observar cuidadosamente o omento menor e o ligamento hepato-gástrico e determinar a presença de uma artéria hepática aberrante, que pode se originar da artéria gástrica esquerda e percorrer o omento em direção ao hilo hepático. Fato de significativa importância, principalmente na atualidade, pela crescente incidência de gastroplastias realizadas. Também se deve examinar a face posterior do pedículo hepático e observar a presença de uma possível artéria hepática di- reita proveniente da artéria mesentérica superior. Segundo Ottone et al. nestes casos ela percorre um trajeto retro duodenal até alcançar o hilo hepático ${ }^{20}$.

Consideramos que o estudo e a pesquisa na disciplina da anatomia humana é uma real necessidade, pois contribui de maneira inequívoca com a prática da medicina em geral em especial da cirurgia com a qual possui uma relação fundamental. Salientamos que a realização de novos estudos trará, sempre, valiosas contribuições para a área da saúde como um todo.

As variações nas artérias hepáticas, incluindo seus ramos, são encontradas com frequência e neste estudo foram de $40 \%$, valor semelhante aos da literatura. A variação mais significativa, observada em 10\% (3 casos), foi a da artéria hepática direita com sua origem na artéria mesentérica superior.

\title{
A B S T R A C T
}

\begin{abstract}
Objectives: to demonstrate the minutiae of hepatic arterial system, the incidence of anatomical variations and to compare data obtained from the literature, thus contributing with students and professionals working in this area. Methods: We prepared 45 corpses at the Department of Anatomy of the Pontifical Catholic University of Paraná, between July 2010 and April 2011, of which group 30 displayed integrity of structures. We analyzed the anatomical variations of the hepatic arteries, their main characteristics, such as origin, course, length and diameter. The overall result was expressed as frequency and percentage of cadavers with anatomic variations of the hepatic arterial system. The estimative of this percentage was done by constructing a confidence interval of $95 \%$. Results: There was some kind of anatomical variation in $40 \%(n=12)$ of cadavers. We found 02 variations in the common hepatic artery, 03 in the gastroduodenal artery, 03 in the right hepatic artery, 01 in the left hepatic artery, 01 in the right gastric artery, and 02 in cystic artery. As for the celiac artery, there were variations in length, diameter and height in its origin, which was common on the aorta. The variation of right hepatic artery originating from the superior mesenteric artery was found in $10 \%$ ( $n=$ 3) of the specimens studied and it was considered the most prevalent type of variation in this study. Conclusion: Changes in hepatic arteries are frequently found and in this study their incidence was 40\%, similar to the literature. The most significant change, observed in 10\% (3 cases), was the right hepatic artery with its origin in the superior mesenteric artery.
\end{abstract}

Key words: Hepatic artery. Liver. General surgery. Anatomy. Cadaver.

\section{REFERENCIAS}

1. Haller A. Methodus studi medici. In: Rio-Branco P. Essaisur I'anatomie ET La médecine óperatoire Du tronc coeliaque et de sés branches de I'ártère hépatique em particulier. Paris: G. Steinheil; 1912.

2. Testut L, Latarget A. Tratado de anatomia humana. 9a ed. Barcelona: Salvat; 1968.

3. Moore KL, Arthur FD, Anne MRA. Anatomia Orientada para a Clínica. $6^{a}$ ed. Rio de Janeiro: Elsevier; 2010.

4. Sobotta J. Atlas de Anatomia Humana. $22^{\mathrm{a}}$ ed. Rio de Janeiro: Guanabara Koogan; 2008.

5. Netter FH. Atlas de Anatomia Humana. $5^{a}$ ed. Rio de Janeiro: Elsevier; 2011.

6. Michels NA. Newer anatomy of the liver and its variant blood supply and collateral circulation. Am J Surg. 1966;112(4):337-47.

7. Soin AS, Friend PJ, Rasmussen A, Saxena R, Tokat $Y$, Alexander GJ, et al. Donor arterial variations in liver transplantation: management and outcome of 527 consecutive grafts. Br J Surg. 1996;83(4):637-41.

8. Bertevello PL, Chaib E. Variações do sistema arterial hepático e sua aplicabilidade na bipartição do fígado. Arq gastroenterol. 2002;39(2):81-5.
9. Soares RV, Coelho JCU, Matias JEF, Zeni Neto C, Freitas ACT, Godoy JL. Anatomia da artéria hepatica em doadores e receptors de transplante hepático intervivos. Rev Col Bras Cir. 2006;33(2):637.

10. Michels NA. Variational anatomy of the hepatic, cystic, and retroduodenal arteries; a statistical analysis of their origin, distribution, and relations to the biliary ducts in two hundred bodies. AMA Arch Surg. 1953;66(1):20-34

11. Hiatt JR, Gabbay J, Busuttil RW. Surgical anatomy of the hepatic arteries in 1000 cases. Ann Surg. 1994;220(1):50-2.

12. Chaib E. Transplante de fígado: alterações da artéria hepática e do fígado em 80 doadores. Arq gastroenterol. 1993:30(4):82-7.

13. Kemeny MM, Hogan JM, Goldberg DA, Lieu C, Beatty JD, Kokal $W$, et al. Continuous hepatic artery infusion with an implantable pump; problems with hepatic artery anomalies. Surgery. 1986;99(4):501-4.

14. Daseler EH, Anson BJ, Hambley WC, Reimann AF. The cystic artery and constituents of the hepatic pedicle; a study of 500 specimens. Surg Gynecol Obstet. 1947;85(1):47-63.

15. Freitas ACT, Coelho JCU, Matias JEF, Zeni Neto C, Martins EL, Druszcz CC. Anatomia arterial hepática: estudo em 150 transplantes hepáticos. Rev Col Bras Cir. 2001;28(1):13-6. 
16. Starzl TE, Marchioro TL, Vonkaulla KN, Hermann G, Brittain RS, Waddell WR. Homotransplantation of the liver in humans. Surg Gynecol Obstet. 1963;117:659-76.

17. Suzuki T, Nakayasu A, Kawabe K, Takeda H, Honjo I. Surgical significance of anatomic variations of the hepatic artery. Am J Surg. 1971;122(4):505-12.

18. Pichlmayr R, Ringe B, Gubernatis G, Hauss J, Bunzendahl H Transplantation einer spenderleber auf zweiempfanger (splittingtransplantation), eine neue methodein der weiterentwicklung der leber segment transplantation. Langenbecks Arch Chir. 1988;373(2):127-30.

19. Couinaud C, Houssin D. Controlled partition of the liver for transplantation. Anatomical limitation. Paris: Couinaud C, Houssin D pers. ed. 1991. Analysis of the anatomical difficulties of bipartition; v.1.

20. Ottone NE, Arrotea Molina A, Domínguez ML, Lo Tartaro M, García de Quiros N, Medan C, et al. Arterias hepáticas aberrantes: estudio en 64 cadáveres disecados. Int j morphol. 2006;24(4):581-5.
Recebido em 15/07/2012

Aceito para publicação em 30/08/2012

Conflito de interesse: nenhum

Fonte de financiamento: nenhuma

\section{Como citar este artigo:}

Sebben GA, Rocha SL, Sebben MA, Parussolo Filho P, Gonçalves BHH. Variações da artéria hepática: estudo anatômico em cadáveres. Rev Col Bras Cir. [periódico na Internet] 2013;40(3). Disponível em URL: http://www.scielo.br/rcbc

\section{Endereço para correspondência:}

Geraldo Alberto Sebben

E-mail: sebben2gea@hotmail.com 